\section{LONG-TERM FOLLOW-UP AFTER NEONATAL COLONIZATION WITH EXTENDED SPECTRUM BETA-LACTAMASE GRAM-NEGATIVE BACTERIA}

\author{
K. Kokk ${ }^{1}$, B. Jonsson ${ }^{2}$, O. Aspevall ${ }^{3}$, A. Iversen ${ }^{4}$, \\ C.G. Giske ${ }^{4}$, M. Norman ${ }^{5}$, L. Navér ${ }^{5}$
}

${ }^{1}$ Department of Neonatology, Karolinska University Hospital, ${ }^{2}$ Department of Woman and Child Health,

${ }^{3}$ Department of Laboratory Medicine, Division of

Clinical Microbiology, ${ }^{4}$ Department of Microbiology,

Tumor and Cell Biology (MTC), ${ }^{5}$ Department of

Clinical Science, Intervention and Technology

(CLINTEC)/Division of Pediatrics, Karolinska

Institutet, Stockholm, Sweden

Objective: Neonatal colonization of the gut with multiresistant (ESBL= Extended spectrum betalactamase) gram-negative bacteria has become more common in recent years. The aim of this study was to investigate whether neonatal ESBL colonization becomes long-lasting or disappears.

Methods: Follow-up study of a cohort (13 infants, 7 boys, 10 preterm) born 2008-2009 in Stockholm and colonized with the same clone of Klebsiella pneumoniae-ESBL, as determined by PFGE, during neonatal intensive care. Four children were co-colonized with E.coli-ESBL. Stool cultures were performed every second month after hospital discharge and are planned until 2 years of age or until 3 subsequent negative cultures. Here we report results at follow-up fourteen months after discharge.

Results: Six months after discharge, 9 out of 12 sampled infants were positive for K.pneumoniaeESBL. The corresponding proportions with K.pneumoniae-ESBL-colonization at 8, 10, 12 and 14 months after discharge were $5 / 12,5 / 12$, $4 / 10$ and $2 / 7$ infants. In addition, at follow-up 6 , 12 and 14 months post-discharge, 7/12, 1/10 and $0 / 7$ infants were found to be positive for E.coliESBL. Up to 14 months of follow-up, 4 children had 3 or more consecutive cultures, negative for both K.pneumoniae- and E.coli-ESBL, and thus regarded as probably free from colonization. During follow-up, one infant was treated for a lower urinary tract infection caused by K.pneumoniae-ESBL. No other infection caused by ESBL-producing bacteria occurred.

Conclusion: Neonatal colonization with ESBLproducing gram-negative bacteria appears to be a transient condition. No infant suffered from serious infection caused by ESBL-producing bacteria during the first 14 months of follow-up.

\section{2}

\section{BIOFILM AND ATTACHMENT CAPABILITIES OF STAPHYLOCOCCUS EPIDERMIDIS STRAINS ISOLATED FROM NEWBORN INFANTS}

\author{
E. Hell ${ }^{1}$, K. Hultenby ${ }^{2}$, G. Marchini ${ }^{1}$ \\ ${ }^{1}$ Dept. Women's and Children's Health, Neonatal \\ Unit, ${ }^{2}$ Dept. Laboratory Medicine, Karolinska \\ Insitutet, Stockholm, Sweden
}

Background: Staphylococcus epidermidis is the most commonly isolated etiological agent of nosocomial infections in preterm infants and the main cause of device-related infections. Biofilm formation is its best known virulence factor.

Methods: S. epidermidis strains were isolated from normal skin and blood of preterm infants. Biofilmand initial attachment assays were determined microplates by crystal violet staining as well as on central venous catheter (CVC) visualised by transmission electron microscopy (TEM). Biofilmassociated genes, and the insertion sequence element IS256 were detected by polymerase chain reaction. The autolysin/adhesin atlE genes of isolates were sequenced.

Results: TEM revealed fimbrial structures on the bacterial surface involved at the initial attachment to CVC of a skin isolate. Similarity in the attachment of the isolates was confirmed by cluster analysis of the atIE gene sequences. Biofilm-positive phenotype was a frequent finding in skin but not in blood isolates. No correlation was found between biofilm formation and presence or absence of different biofilm related genes. 9 from 10 blood isolates found to be invasive based on the presence of IS256.

Conclusions: Although biofilm formation is a well known virulence factor of $S$. epidermidis, invasive strains may use an other strategy to be in hiding from the immune system. Since IS256 may affect the expression of certain genes and also may be associated with the antibiotic resistance, these can increase the fitness of invasive strains to cause sepsis in preterms. 